\title{
ICEBERG OSCILLATIONS AND OCEAN WAVES
}

\author{
by
}

\section{Peter Schwerdtfeger}

(Flinders Institute of Atmospheric and Marine Sciences, Flinders University of South Australia, Bedford Park, South Australia 5042, Australia)

\section{ABSTRACT}

The frequencies of both linear and angular oscillations in a vertical plane of a floating iceberg are shown to converge as the horizontal dimensions become relatively larger. For icebergs of thickness of $250 \mathrm{~m}$, the calculated period of about $30 \mathrm{~s}$ is supported by actual observations which have been reported. Because swe11 in the Southern Ocean may extend to such long periods, a critical relationship between iceberg dimensions and ocean wavelengths has been formulated. This relates the conditions under which the length and natural period of an iceberg may, respectively, coincide with the wavelength and wave period of the ocean.

\section{INTRODUCTION}

An iceberg in the sea has two principal oscillatory modes, both in a vertical plane. The first of these is a simple linear oscillation, and may be described as "bobbing", while the other is an angular oscillation about the centre of mass, and may be described as "rocking". Both of these motions are, of course, damped, but, in an ocean where wave action may include frequencies close to those natural to a particular iceberg, the latter may acquire a considerable amount of energy and suffer from the resultant stresses. In the following discussion, the icebergs will be restricted to those of rectangular vertical cross-section.

\section{LINEAR OSCILLATIONS OR "BOBBING"}

Referring to Figure 1, it is seen that if a cylindrically-shaped iceberg of any horizontal cross-section suffers a transient depression from its equilibrium flotation level in the sea, then, if the effects of damping are disregarded as having a significant influence on the frequency of subsequent oscillations, the period of these oscillations, $T_{b}$, is given by:

$$
T_{\mathrm{b}}=2 \pi\left(\frac{\rho_{\mathrm{i}} h}{\rho_{\mathrm{W} g}}\right)^{0.5}
$$

where $h$ is the total height or thickness of the iceberg, $g$ is the acceleration caused by gravity, and $\rho_{i}$ and $\rho_{w}$ are the densities of the ice and sea-water respectively.

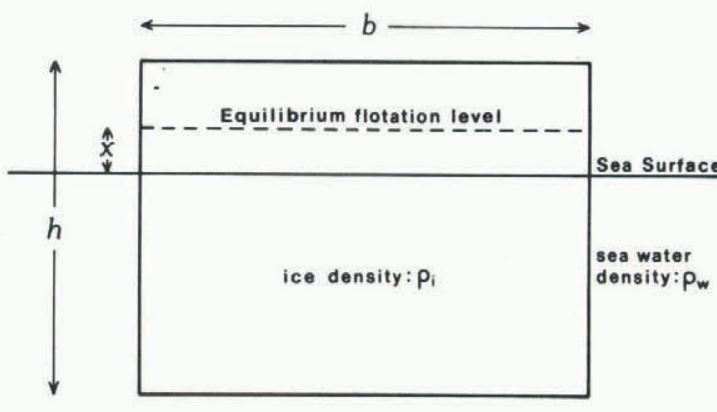

$$
\ddot{x}=-\frac{\rho_{w} g}{\rho_{i} h} x \quad T_{b}=2 \pi \sqrt{\frac{\rho_{i} h}{\rho_{w} g}}
$$

Fig.1. Vertical oscillation ("bobbing") of i cebergs.

Using values of 0.9 and $1.018 \mathrm{Mg} \mathrm{m}^{-3}$ for $\rho_{i}$ and $\rho_{w}$ as well as $9.81 \mathrm{~m} / \mathrm{s}^{-2}$ for $g$, Equation (1.1) becomes:

$$
T_{\mathrm{b}}=1.89 h^{0 \cdot 5} \text {. }
$$

2. ROTATIONAL OSCILLATIONS OR "ROCKING"

Figure 2 shows an iceberg of rectangular cross-section which has suffered a small angular displacement $\theta$ from its normal position of horizontal equilibrium. The centre of mass does not move in this pure oscillation in the vertical plane in which it has been assumed, for simplicity, that the iceberg is a right-angled parallelepiped, i.e. all vertical and horizontal cross-sections are rectangular. The moment of inertia about the centre of mass in the plane of the diagram in Figure 2 is:

$$
\frac{1}{12} \rho_{i} \ln b\left(h^{2}+b^{2}\right)
$$

where $b$ is the breadth and $l$ is the length of the iceberg, not shown in the diagram. 


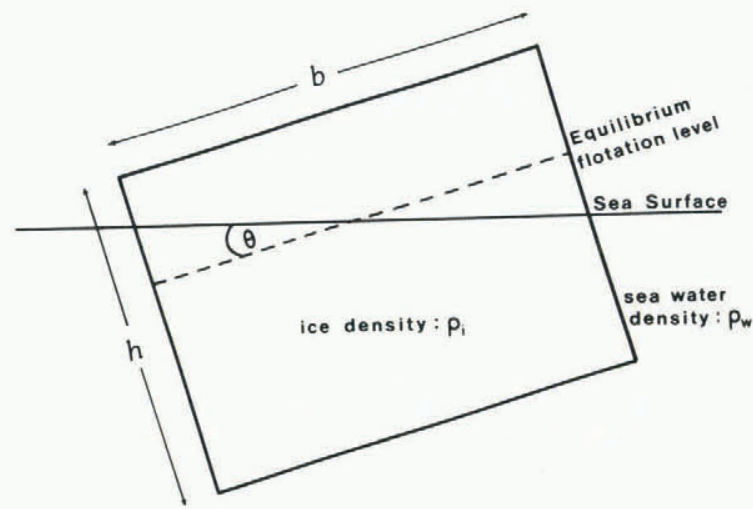

$\ddot{\theta}=\frac{-p_{w} g b^{2}}{p_{i} h\left(h^{2}+b^{2}\right)} \theta \quad T_{r}=2 \pi \sqrt{\frac{p_{i} h\left(h^{2}+b^{2}\right)}{p_{w} g b^{2}}}$

Fig.2. Rotational oscillation ("rocking") of icebergs.

The period of small, rocking oscillations is easily shown to be:

$$
T_{\mathrm{r}}=2 \pi\left(\frac{\rho_{\mathrm{i}} h\left(h^{2}+b^{2}\right)}{\rho_{\mathrm{w}} g b^{2}}\right)^{0.5}
$$

It follows from Equations (1.1) and (2.1) that as $b \rightarrow \infty, T_{\mathrm{r}} \rightarrow T_{\mathrm{b}}$ : a not unexpected result. Again, substituting the same values as before, Equation (2.1) becomes:

$$
T_{\mathrm{r}}=1.89\left(\frac{h\left(h^{2}+b^{2}\right)}{b^{2}}\right)^{0.5}
$$

3. DEPENDENCE OF OSCILLATIONS ON DIMENSIONS

If the icebergs discussed above are described by a height to breadth ratio $\alpha$, such that $b=\alpha h$, then:

$$
T_{\mathrm{r}}=1.89\left(\frac{1+\alpha^{2}}{\alpha^{2}}\right)^{0.5}(h)^{0.5} \text {, }
$$

from which equation it is readily seen that for icebergs with $\alpha>3, T_{\mathrm{r}}$ and $T_{\mathrm{b}}$ differ by less than $10 \%$. This is shown in Figure 3 , which is restricted to a range of realistic values of $h$ and $b$. It is interesting to note that the maximum period for "natural" oscillations in icebergs of about. $30 \mathrm{~s}$ is determined by the maximum value of $h$ encountered being about $250 \mathrm{~m}$. Actual observations on icebergs in the Southern Ocean appear to support this conclusion, (Foldvik and others 1980). Any iceberg with $a \leqslant 1$ is, of course, unstable when afloat.

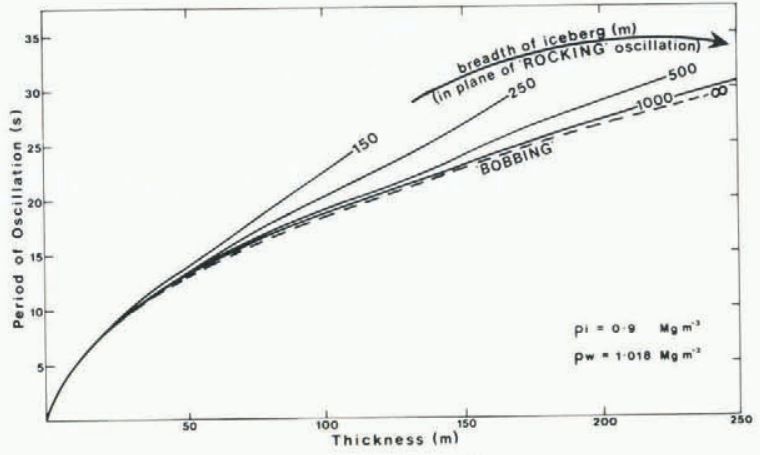

Fig.3. Oscillatory motion of icebergs of rectangular cross-section.

4. OCEAN WAVES

A standard result of hydrodynamic wave theory linking the velocity of propagation $c$ of surface water waves and $\lambda$ their length with $H$, the depth of the water, is:

$$
c^{2}=\frac{g \lambda}{2 \pi} \tanh \left(\frac{2 \pi H}{\lambda}\right),
$$

in which expression the density of air, when compared to that of sea-water, has been neglected.

For any water deep enough to support an iceberg, $\tanh \frac{2 \pi H}{\lambda} \approx 1$ and Equation (4.1) becomes:

$$
c^{2}=\frac{g \lambda}{2 \pi},
$$

and since the period of the waves is given by

$$
T=\frac{\lambda}{c}
$$

it follows that

$$
c=\frac{g}{2 \pi} T
$$

and

$$
\lambda=\frac{g}{2 \pi} T^{2} .
$$

With m.k.s. units, this expression further simplifies to:

$$
c=1.56 T \text { and } \lambda=1.56 T^{2} \text {. }
$$

Figure 4 shows the graphical relationship between the period and wavelength of surface waves.

\section{RESPONSE OF ICEBERGS TO OCEAN WAVES}

The natural frequency of icebergs will coincide with that of the surrounding waves under certain conditions in which it is certain under certain conditions of amplitude of oscillation of the iceberg will be maximal. When the further condition of ocean wavelength coinciding with the breadth is added, the most likely circumstances for iceberg fracture are provided. 


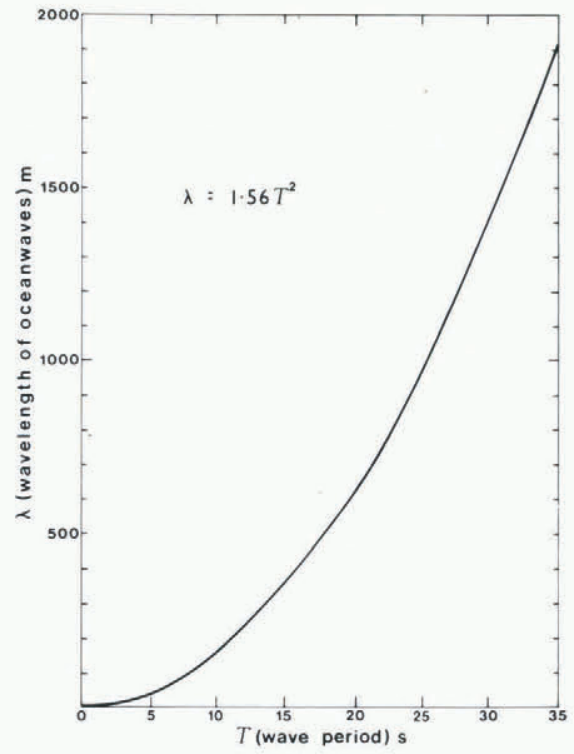

Fig.4. Relationship between the period and wave length of surface waves.
It. seems clear that more information on frequencies and amplitudes of swell in the Southern Ocean is required, not only to elucidate a possibly significant natural mode of iceberg disintegration but also to allow for the most advantageous selection of iceberg dimensions, should iceberg towing become a reality.

\section{REFERENCE}

Foldvik A, Gammelsr $\phi$ d T, Gjessing Y 1980 Measurements of oscillations and flexure of icebergs. Annals of Glaciology 1:
The "bobbing" period is taken as being the most characteristic since it has been shown to correspond relatively closely to the "rocking" period for most real icebergs.

From Equations (1.1) and (4.3) it follows that when the period of ocean waves coincides with the natural frequency of the iceberg being supported, the wavelength and the thickness of the iceberg are related by:

$$
\lambda=2 \pi \frac{\rho_{i}}{\rho_{\mathrm{w}}} h .
$$

If, further, the oceanic wavelength becomes equal to the iceberg width, then $\lambda=b=\alpha h$, so that the critical width-to-thickness ratio of an iceberg follows as:

$$
\alpha=\frac{2 \pi \rho_{i}}{\rho_{\mathrm{W}}} .
$$

It is important to note that Equation (5.2) is independent of actual iceberg dimensions and, with the values of the densities used before, becomes: $\alpha=5.55$. With these same values, Equation (5.1) simplifies to: $\lambda=5.55 h$.

There is a lack of data on wave periodicities in the Southern Ocean, but waves of significant amplitudes of periods of up to $20 \mathrm{~s}$ and hence wavelengths of $624 \mathrm{~m}$ seem likely. Under such oceanic conditions, parallelepipedal icebergs with similar horizontal dimensions and overall thicknesses of about $112 \mathrm{~m}$ might be expected to be particularly susceptible to stresses induced by oscillations. 